\title{
BIOMASSA E ESTOQUE DE CARBONO E NUTRIENTES EM FLORESTAS MONTANAS DA MATA ATLÂNTICA NA REGIÃO NORTE DO ESTADO DO RIO DE JANEIRO(1)
}

\author{
Gláucio de Mello Cunha ${ }^{(2)}$, Antonio Carlos Gama-Rodrigues ${ }^{(3)(4)}$, Emanuela \\ Forestieri Gama-Rodrigues ${ }^{(3)}$ \& Ary Carlos Xavier Velloso ${ }^{(3)}$
}

\begin{abstract}
RESUMO
Os estudos sobre balanço de $\mathrm{C}$ e nutrientes em florestas naturais permitem avaliar possíveis alterações decorrentes de técnicas de manejo aplicadas e possibilitam inferir a sustentabilidade dessas florestas. Os objetivos deste trabalho foram avaliar o teor de nutrientes de espécies nativas e quantificar a biomassa (parte aérea + serapilheira) e os estoques de $\mathrm{C}$ e nutrientes em fragmentos florestais montanos da Mata Atlântica (Floresta Ombrófila Densa Montana) na região norte do Estado do Rio de Janeiro, no período de maio de 1999 a abril de 2001. Foram selecionados dois fragmentos, localizados a 900 e $600 \mathrm{~m}$ de altitude, na vertente atlântica do Parque Estadual do Desengano, RJ. O solo de ambos os fragmentos florestais foi classificado como Cambissolo Háplico Tb distrófico. O valor médio de biomassa (parte aérea + serapilheira) foi de 166,8 $\mathrm{Mg} \mathrm{ha}^{-1}$. Em consequência, a acumulação média de $\mathrm{C}$ na vegetação foi de $67,2 \mathrm{Mg} \mathrm{ha}^{-1}$. Os estoques médios de $\mathrm{N}$, $\mathrm{P}, \mathrm{K}, \mathrm{Ca}$ e Mg na vegetação foram de 1.152, 44,4, 276,5, 603,5 e 127,9 $\mathrm{kg} \mathrm{ha}^{-1}$, respectivamente. Por outro lado, as espécies revelaram distinta capacidade de estoque de nutrientes. O balanço negativo de P, K e Ca ssolo - (parte aérea + serapilheira)\} indica que esses elementos constituem principais fatores nutricionais limitantes ao crescimento dos fragmentos florestais montanos da Mata Atlântica na região norte-fluminense.
\end{abstract}

Termos de indexação: balanço de nutrientes, fragmento florestal, solo florestal.

\footnotetext{
(1) Parte da Tese de Doutorado do primeiro autor apresentada ao Curso de Pós-Graduação em Produção Vegetal, Universidade Estadual do Norte Fluminense Darcy Ribeiro - CCTA/UENF. Recebido para publicação em novembro de 2008 e aprovado em maio de 2009 .

(2) Professor Assistente Centro de Ciências Agrárias, Universidade Federal do Espírito Santo - UFES. Caixa Postal 16, CEP 29500000 Alegre (ES). E-mail: glaucioml@yahoo.com.br

(3) Professor Associado do Laboratório de Solos do Centro de Ciências e Tecnologias Agropecuárias - CCTA, Universidade Estadual do Norte Fluminense Darcy Ribeiro - UENF. CEP 28013-602 Campos dos Goytacazes (RJ). E-mails: tonygama@uenf.br; emanuela@uenf.br; velloso@uenf.br

(4) Bolsista do CNPq.
} 


\title{
SUMMARY: BIOMASS, CARBON AND NUTRIENT POOLS IN MONTANE ATLANTIC FORESTS IN THE NORTH OF RIO DE JANEIRO STATE, BRAZIL
}

\begin{abstract}
Nutrient balance in tropical forest allows an evaluation of eventual differences due to management practices and conclusions on forest sustainability. The aim of this study was to evaluate the nutrient concentration in native species and quantify biomass production and $C$ and nutrient balance in tropical montane Atlantic Forest fragments (Dense Montane Ombrophile Forest) in the North of Rio de Janeiro State, from May 1999 to April 2001. Two sites were selected in the State Park of Desengano, at 900 and $600 \mathrm{~m}$ asl. The soil of both forest fragments was classified as Dystrophic Haplic Cambissol. The biomass production (above ground + accumulated litter) was $166.8 \mathrm{Mg} \mathrm{ha}^{-1}$. Consequently, the C accumulation was $67.2 \mathrm{Mg} \mathrm{ha}^{-1}$. The mean $\mathrm{N}, \mathrm{P}, \mathrm{K}, \mathrm{Ca}$ and $\mathrm{Mg}$ pools were 1,152, 44.4, 276.5, 603.5 and $127.9 \mathrm{~kg} \mathrm{ha} \mathrm{g}^{-1}$, respectively. However, at each forest site, the species differed in nutrient pools. The negative balance \{soil - (above ground + litter)\} of $P, K$ and $C a$ indicated that these elements were the most limiting to the growth of montane forest fragments at the study sites.
\end{abstract}

Index terms: nutrient balance, forest fragment, forest soil.

\section{INTRODUÇÃO}

Na região norte-fluminense a cobertura natural atual de Mata Atlântica é inferior a $5 \%$ da mata original (CIDE, 2004), devido ao longo período de atividades agropecuárias, especialmente da cana-deaçúcar e do café. Isso resultou não apenas na drástica redução do maciço florestal, mas também na degradação dos solos. Os fragmentos florestais remanescentes são pobres em espécies de alto valor comercial, caracterizando ecossistemas muito degradados quanto ao solo e à biodiversidade (GamaRodrigues \& May, 2001). Nesse contexto, ocorreu a decadência socioeconômica da região, na qual as atividades de uso atual das terras são pastagens também degradadas. Assim, torna-se necessário implantar sistemas de manejo agroflorestal e, ou, florestal que melhorem a qualidade florística e edáfica dos fragmentos florestais remanescentes, a fim de aumentar a renda e proporcionar a sustentabilidade do agronegócio.

Nos solos de baixa fertilidade, os estoques de $\mathrm{C} \mathrm{e}$ nutrientes encontrados na serapilheira e na parte aérea são importantes na definição do balanço de $\mathrm{C} \mathrm{e}$ nutrientes e poderiam servir de indicadores da capacidade produtiva dos diversos fragmentos florestais da Mata Atlântica (Gama-Rodrigues \& Barros, 2002; Gama-Rodrigues et al., 2008). Assim, estudos desse tipo poderiam ainda fornecer subsídios para o desenvolvimento de técnicas de manejo para recuperação e, ou, melhoria dos atuais fragmentos florestais, bem como na recomposição de parte do maciço florestal nas áreas de pastagens degradadas adjacentes (Gama-Rodrigues \& May, 2001).

$\mathrm{O}$ estoque de C no sistema solo-planta em fragmentos de florestas naturais também pode indicar o potencial dessas coberturas em sequestrar $\mathrm{C}$ da atmosfera (Alegre et al., 2004), constituindo, assim, um serviço ambiental promotor da mitigação de mudanças climáticas por gases de efeito estufa, em especial o $\mathrm{CO}_{2}$ (Fernandes, 2006).

Os objetivos deste trabalho foram determinar o teor de nutrientes de espécies nativas e quantificar a biomassa (parte aérea + serapilheira) e os estoques de $\mathrm{C}$ e nutrientes em fragmentos florestais da Mata Atlântica na região montana norte-fluminense.

\section{MATERIAL E MÉTODOS}

O trabalho foi realizado em dois fragmentos florestais de Mata Atlântica, no Parque Estadual do Desengano, município de Santa Maria Madalena, região norte-fluminense, RJ, Brasil ( $21^{\circ} 37^{\prime} \mathrm{S}$ e $42^{\circ}$ $05^{\prime}$ W). O primeiro fragmento florestal (M1), de aproximadamente 200 ha, está localizado a $900 \mathrm{~m}$ de altitude, interligado a um contínuo florestal de 25.000 ha (Brasil, 1983). O outro fragmento florestal (M2) está localizado a $600 \mathrm{~m}$ de altitude, de tamanho aproximado de 10 ha. Ambos os fragmentos estão localizados na vertente atlântica. Os dois fragmentos florestais, de aproximadamente 40 anos de idade, compõem, fitoecologicamente, a Floresta Ombrófila Densa Montana, sendo o substrato geológico local composto por gnaisses-granitoides do Complexo Paraíba do Sul de idade pré-cambriana (Brasil, 1983). O clima foi classificado como Cwa, caracterizado por uma pluviosidade média anual de $1.440 \mathrm{~mm}$, com período seco de maio a agosto. Durante o período de estudo do presente trabalho a pluviosidade foi de $1.783 \mathrm{~mm}$ de maio de 1999 a abril de 2000 , enquanto entre maio de 2000 e abril de 2001 ela ficou em $1.106 \mathrm{~mm}$. O solo dos fragmentos florestais é um Cambissolo Háplico Tb distrófico textura franco-argiloarenosa, em relevo forte ondulado (Embrapa, 1999). A escolha dos fragmentos foi decorrente da facilidade 
de acesso às áreas de estudo, especialmente no período chuvoso, e do estado de conservação deles, com base na presença de um número significativo de árvores com diâmetro (DAP) acima de $10 \mathrm{~cm}$.

Em cada fragmento florestal foram demarcadas três parcelas de $25 \times 20 \mathrm{~m}$, instaladas na encosta no sentido vertical, do topo para a base, distantes cerca de $500 \mathrm{~m}$ da borda no fragmento M1 e $150 \mathrm{~m}$ da borda no M2. Assim, assumiu-se que o efeito de borda não foi significativo na mensuração dos dados (Silva \& Nascimento, 2001). O diâmetro com casca do fuste a 1,3 m (DAP) foi medido com fita métrica em todas as árvores das parcelas no período seco, de maio a agosto de 1999/2000. A altura comercial e total dos indivíduos com DAP maior do que $10 \mathrm{~cm}$ foi medida com vara graduada. Considerou-se como altura comercial a medida da base até a inserção do primeiro galho que forma a copa da árvore. Nas épocas secas e chuvosas de 1999 a 2001 foram realizadas amostragens de folhas verdes maduras e galhos (2 a $5 \mathrm{~cm}$ de diâmetro) na porção intermediária das copas, para análise química. Quando possível, coletou-se também inflorescência para identificação botânica. As amostras de lenho e de casca foram coletadas com auxílio de um trado em $50 \%$ das árvores das classes diamétricas até $25 \mathrm{~cm}$ de DAP, e em todas as árvores com DAP maior do que $25 \mathrm{~cm}$, conforme Gama-Rodrigues (1997).

A estimação do volume das árvores foi feita por modelos testados por Campos et al. (2001). Para o cálculo de volume, as árvores foram separadas em dois grupos distintos, de acordo com a classe diamétrica.

Para árvores com DAP de até $20 \mathrm{~cm}$, a estimativa do volume foi obtida por:

$$
\begin{gathered}
\mathrm{V}=0.00005 . \mathrm{DAP}^{1,84209} \cdot \mathrm{H}^{1,09375} \\
\left(\mathrm{EXP}^{(-2,18395-\mathrm{tx} 1 / \mathrm{dap})}\right)\left(1-\left(\mathrm{tx}_{2}-\mathrm{Hc} / \mathrm{DAP}\right)^{0,18813}\right)
\end{gathered}
$$

Para árvores com DAP superior a $20 \mathrm{~cm}$, essa estimativa foi obtida por:

$$
\begin{gathered}
\mathrm{V}=0.00013 . \mathrm{DAP}{ }^{1,81620} \cdot \mathrm{H}^{0,77344} \\
\left(\operatorname{EXP}^{(-7,37223 \cdot \operatorname{tx} 1 / \mathrm{dap})}\right)\left(1-\left(\mathrm{tx}_{2}-\mathrm{Hc} / \mathrm{DAP}\right)^{0,32428}\right)
\end{gathered}
$$

em que $\mathrm{V}$ é o volume em $\mathrm{m}^{3}$; DAP, o diâmetro à altura do peito; $\mathrm{H}$, a altura total; $\mathrm{Hc}$, a altura comercial; e $\mathrm{tx}_{1}$ e tx $\mathrm{t}_{2}$, variáveis binárias. Assim, a combinação $0 \mathrm{e}$ 0 para tx $\mathrm{e}_{1}$ e tx ${ }_{2}$ resulta em volume com casca do tronco, enquanto a combinação 1 e 0 resulta em volume sem casca e a combinação 0 e 1 resulta em volume de galhos (vg).

$\mathrm{Na}$ estimativa da biomassa foram considerados valores médios de densidade de madeira (galho e lenho) de 0,54 e $0,45 \mathrm{~kg} \mathrm{~m}^{-3}$ para casca, respectivamente, obtidos para espécies nativas da Mata Atlântica, conforme Clevelario Junior (1996). Para a biomassa de folhas, empregou-se a relação proposta por Grubb (1977) entre a quantidade de folhas na copa (P) e a queda anual destas $(\mathrm{Q})(\mathrm{P} / \mathrm{Q}=1,25)$, utilizada também por Clevelario Junior (1996) no balanço de nutrientes de um ecossistema florestal tropical baixo-montano.
A amostragem de serapilheira acumulada sobre o solo foi realizada em duas épocas do ano (seca e chuvosa de 1999/2001), com quatro repetições aleatórias por parcela, utilizando-se um quadrado de madeira de $0,25 \mathrm{~m}^{2}$. Em ambas as épocas de amostragem foram determinados, nos componentes das árvores (folha, galho, casca e lenho) e na serapilheira, após secagem em estufa a $65^{\circ} \mathrm{C}$ até peso constante, os teores de $\mathrm{K}$ (por fotometria de chama), de $\mathrm{P}$ (colorimetricamente, pelo método do complexo fosfomolíbdico, reduzido com ácido ascórbico, modificado por Braga \& Defelipo, 1974), de Ca e Mg (por espectrofotometria de absorção atômica, após digestão nítrico-perclórica) e de $\mathrm{N}$ (pelo método Kjeldahl, descrito por Bataglia et al., 1983). $\mathrm{O} \mathrm{C}$ foi determinado por oxidação com $\mathrm{K}_{2} \mathrm{Cr}_{2} \mathrm{O}_{7} \mathrm{em}$ meio ácido, como descrito por Anderson \& Ingram (1996). A quantidade de C e nutrientes nos componentes das árvores e da serapilheira foi estimada pelo produto entre o teor e a matéria seca.

Em cada parcela foi coletada uma amostra composta de solo, proveniente de quatro amostras simples, nas profundidades de 0 a 20, 20 a 40, 40 a 60 e 60 a $80 \mathrm{~cm}$, na época seca de 2000 (maio a agosto), para análise de $\mathrm{pH}$ (água); $\mathrm{P}$ e $\mathrm{K}$ extraíveis, por Mehlich-1; Ca e Mg trocáveis, por $\mathrm{KCl} 1 \mathrm{~mol} \mathrm{~L}^{-1}$; C orgânico, por oxidação com $\mathrm{K}_{2} \mathrm{Cr}_{2} \mathrm{O}_{7}$; e N total do solo, pelo método Kjeldahl (Embrapa, 1997).

Para o balanço interno de nutrientes, tomaram-se valores dos conteúdos dos nutrientes no solo (em diferentes profundidades), na serapilheira acumulada e na parte aérea das árvores, para cada fragmento florestal (Gama-Rodrigues et al., 2008). O balanço interno de nutrientes foi obtido pela seguinte expressão:

$$
\begin{gathered}
\text { Balanço Interno de Nutrientes }=\text { solo - } \\
\text { (parte aérea + serapilheira) }
\end{gathered}
$$

Desse modo, o balanço interno de nutrientes expressa a diferença de estoque entre o solo e a vegetação aérea.

$\mathrm{Na}$ análise dos dados adotou-se a estatística descritiva (média, desvio-padrão, coeficiente de variação, valor máximo e mínimo). Os dados de teor de nutrientes na folha foram submetidos à análise de componentes principais, que possibilita sintetizar a variação multidimensional dos dados analisados em um diagrama, ordenando-os nos eixos, de acordo com suas similaridades em torno das variáveis utilizadas (Ter Braak, 1986). No presente trabalho considerouse o autovetor < 0,70 como de baixa associação para interpretação dos componentes principais (Wick et al., 1998). Além disso, foram usados apenas os dois primeiros componentes, considerados suficientes para explicar os dados e pela facilidade de interpretação de um gráfico em duas dimensões (Gomes et al., 2004; Barreto et al., 2008). Cada fragmento florestal foi considerado um tratamento de efeito fixo (Lugo et al., 1990; Gama-Rodrigues et al., 2007). 


\section{RESULTADOS E DISCUSSÃO}

\section{Dendometria e biomassa}

Nos dois fragmentos florestais, o maior número de indivíduos ocorreu na classe diamétrica de 10 a $14 \mathrm{~cm}$, o que correspondeu a $46 \%$ no M1 e $35 \%$ no M2, em relação ao número total de indivíduos (Figura 1). Borém (1998) encontrou aproximadamente $20 \%$ dos indivíduos nessa mesma classe diamétrica em um fragmento de Mata Atlântica submontana, na região centro-norte do Rio de Janeiro. No M2, ainda, verificou-se maior número de classes diamétricas, porém foi maior a ausência de indivíduos em algumas classes (Figura 1). Esses resultados sugerem algum problema na regeneração, que pode estar associado com possíveis cortes seletivos ocorridos no decorrer dos últimos anos, como indicado pela presença de troncos cortados nesse fragmento.

Nos fragmentos florestais estudados, foram observados valores médios de $20,53 \mathrm{~cm}$ para DAP, $30,19 \mathrm{~m}^{2} \mathrm{ha}^{-1}$ de área basal, densidade de 830 indivíduos ha-1 e biomassa áerea total de 158,14 Mg ha-1 (Quadro 1). Moreno (1998), em dois fragmentos de encosta da Mata Atlântica, localizados também no Parque Estadual do Desengano, encontrou área basal média de $38 \mathrm{~m}^{2} \mathrm{ha}^{-1}$.

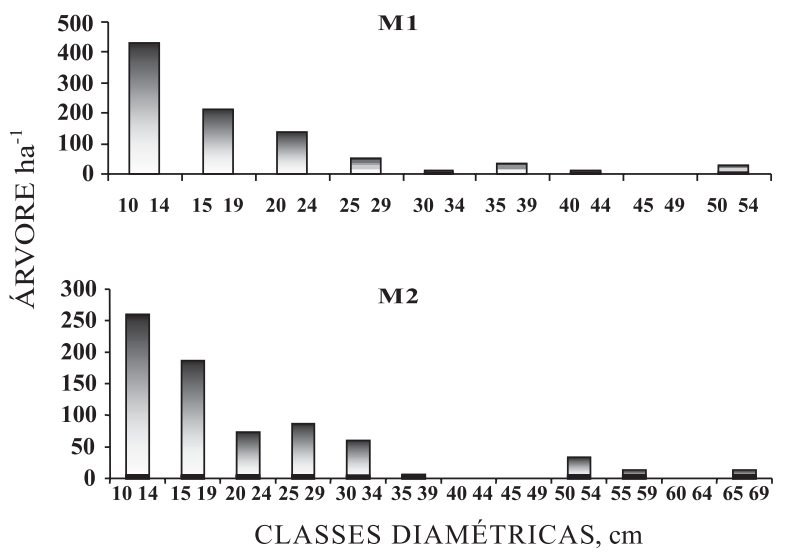

Figura 1. Distribuição do número de árvores por hectare e por classe diamétrica de indivíduos com DAP maior do que $10 \mathrm{~cm}$ em fragmentos florestais (M1 e M2) da Mata Atlântica, na região norte-fluminense.
A partição da biomassa total em lenho, galho, casca e folha foi de $61,21,13$ e $5 \%$, respectivamente, no fragmento M1, enquanto no M2, na mesma ordem, foi de 61, 25, 10 e $4 \%$ (Quadro 1). Em fragmento de Mata Atlântica submontano, no Rio de Janeiro, a biomassa de galhos e cascas correspondeu, respectivamente, a 27 e $6 \%$ da biomassa total (Clevelario Júnior, 1996). Em outros tipos florestais, os valores de biomassa de galhos em relação à biomassa total foram de $14 \%$ em floresta montana (Grimm \& Fassbender, 1981).

Os valores de biomassa aérea total dos fragmentos florestais estudados estão dentro da faixa de variação de biomassa geralmente encontrada na Mata Atlântica, sobretudo porque no presente trabalho só foram considerados, como estimativa, aqueles indivíduos com DAP maior do que $10 \mathrm{~cm}$ (Quadro 1). Clevelario Júnior (1996) encontrou biomassa aérea total de $371 \mathrm{Mg}$ ha $^{-1}$ de árvores com DAP acima de $5 \mathrm{~cm}$ de fragmentos florestais submontanos, no Estado do Rio de Janeiro. No entanto, em outro fragmento florestal submontano, na região do Médio Rio Doce, MG, a biomassa aérea foi de 111,6 $\mathrm{Mg} \mathrm{ha}^{-1}$ (Drumond, 1996).

As médias de diâmetro, altura total e altura comercial e suas respectivas variações, bem como as estimativas de volume e de biomassa, são mostradas no quadro 2. Verifica-se que praticamente não houve diferença entre os dois fragmentos florestais para altura total e comercial. No M2, de maneira geral, houve maior variabilidade (indicada pelo desviopadrão) em todas as variáveis de volume e biomassa do que no M1.

\section{Balanço de nutrientes}

As espécies de ambos os fragmentos florestais revelaram grande variação de teor de nutrientes nas folhas (Quadro 3). No fragmento M1, o maior teor de $\mathrm{N}$ foi verificado na espécie Phytolacca dioica; de $\mathrm{P}$, em Sorocea bonplandii; de K, em Virola oleifera; de Ca, numa espécie não identificada da família Lauraceae, no valor de 19,56 $\mathrm{g} \mathrm{kg}^{-1}$; e de $\mathrm{Mg}$, em Sorocea bonplandii (Quadro 3). No fragmento M2, o maior teor de $\mathrm{N}$ foi observado na espécie Hyeronima alchorneoides; de P, em Campomanesia sp.; de K, em duas espécies não identificadas das famílias Anacardiaceae e Melastomataceae, no valor de

Quadro 1. Diâmetro à altura do peito (DAP), área basal (AB), densidade de indivíduos e produção de matéria seca dos componentes das árvores amostradas em fragmentos florestais (M1 e M2) da Mata Atlântica, na região norte-fluminense

\begin{tabular}{|c|c|c|c|c|c|c|c|c|}
\hline Fragmento & DAP & AB & Densidade & Lenho & Casca & Galho & Folha & Total \\
\hline & $\mathrm{cm}$ & $\mathrm{m}^{2} \mathrm{ha}^{-1}$ & Ind. ha ${ }^{-1}$ & & & $\mathrm{Mg} \mathrm{ha}^{-1}$ & & \\
\hline M1 & 19,35 & 33,25 & 927 & 90,51 & 19,72 & 31,47 & 6,71 & 148,41 \\
\hline M2 & 21,71 & 27,13 & 733 & 102,59 & 17,05 & 41,82 & 6,40 & 167,86 \\
\hline
\end{tabular}


Quadro 2. Estatística descritiva de diâmetro (DAP), altura total (HT), altura comercial (HC), volumes de galho, lenho, casca e total e matéria seca de galho, lenho e casca das árvores amostradas em fragmentos florestais (M1 e M2) da Mata Atlântica, na região norte-fluminense

\begin{tabular}{|c|c|c|c|c|c|c|c|c|c|c|}
\hline & \multirow{2}{*}{ DAP } & \multirow{2}{*}{ HT } & \multirow{2}{*}{$\mathrm{HC}$} & \multicolumn{4}{|c|}{ Volume } & \multicolumn{3}{|c|}{ Matéria seca } \\
\hline & & & & Galho & Lenho & Casca & Total & Lenho & Galho & Casca \\
\hline & \multirow[t]{2}{*}{$\mathrm{cm}$} & \multicolumn{2}{|c|}{$-\mathrm{m}-$} & \multicolumn{4}{|c|}{$-m^{3}$} & \multicolumn{3}{|c|}{ - $\mathrm{kg}$} \\
\hline & & & & \multicolumn{4}{|c|}{ M1 } & & & \\
\hline $\mathrm{N}^{\circ}$ indiv. ${ }^{(1)}$ & 139 & 139 & 139 & 139 & 139 & 139 & 139 & 139 & 139 & 139 \\
\hline Mínimo & 10,04 & 6,90 & 4,30 & 0,0066 & 0,0244 & 0,0059 & 0,0369 & 13,2 & 3,5 & 3,2 \\
\hline Máximo & 53,48 & 20,50 & 15,50 & 0,6126 & 1,4635 & 0,2184 & 2,2945 & 790,3 & 330,8 & 117,9 \\
\hline Média & 19,35 & 10,71 & 8,02 & 0,0629 & 0,1809 & 0,0394 & 0,2832 & 97,7 & 34,0 & 21,3 \\
\hline \multirow[t]{2}{*}{$\mathrm{DP}$} & 8,93 & 2,85 & 2,48 & 0,1019 & 0,2470 & 0,0481 & 0,3920 & 133,4 & 55,0 & 26,0 \\
\hline & \multicolumn{10}{|c|}{$\mathrm{M} 2$} \\
\hline No indiv. & 110 & 110 & 110 & 110 & 110 & 110 & 110 & 110 & 110 & 110 \\
\hline Mínimo & 10,19 & 4,70 & 3,40 & 0,0009 & 0,0089 & 0,0039 & 0,0137 & 4,8 & 0,5 & 1,7 \\
\hline Máximo & 68,44 & 20,60 & 15,80 & 1,0292 & 2,4016 & 0,2732 & 3,7040 & 1296 & 555,8 & 147,5 \\
\hline Média & 21,71 & 10,53 & 7,48 & 0,1056 & 0,2597 & 0,0513 & 0,4166 & 140,2 & 57,0 & 23,3 \\
\hline $\mathrm{DP}$ & 12,71 & 3,60 & 2,93 & 0,1964 & 0,4327 & 0,0640 & 0,6865 & 233,7 & 106,1 & 30,2 \\
\hline
\end{tabular}

${ }^{(1)} \mathrm{N}^{o}$ indiv.: número de indivíduos com DAP maior do que $10 \mathrm{~cm}$; DP: desvio-padrão.

19,23 $\mathrm{g} \mathrm{kg}^{-1}$; de Ca, também em espécie não identificada da família Melastomataceae, no valor de $18,23 \mathrm{~g} \mathrm{~kg}^{-1}$; e de $\mathrm{Mg}$, em Geissospermum laevis (Quadro 3). Os teores médios de N, P, K, Ca e Mg na folha das espécies do fragmento M1 foram de 26,66, 2,24, 16,73, 9,08 e 3,28 $\mathrm{g} \mathrm{kg}^{-1}$, respectivamente; no M2, foram de 25,80 $\mathrm{g} \mathrm{kg}^{-1}$ de $\mathrm{N}, 0,94 \mathrm{~g} \mathrm{~kg}^{-1}$ de $\mathrm{P}$, $6,32 \mathrm{~g} \mathrm{~kg}^{-1}$ de K, 9,78 $\mathrm{g} \mathrm{kg}^{-1}$ de Ca e 2,52 $\mathrm{g} \mathrm{kg}^{-1}$ de Mg. Por outro lado, no fragmento M1 a maior variação de teores entre as espécies foi do P (CV $66 \%$ ), seguido de $\mathrm{Ca}(49 \%), \mathrm{Mg}$ (45\%), K (43\%) e N (25\%). Contudo, no fragmento M2 a maior variação ocorreu na concentração de K (124\%), seguido de Mg (72 \%), P (33\%), Ca (30 \%) e N (25\%).

Esses resultados indicam que há capacidade diferenciada das espécies nativas em absorver nutrientes do solo. Assim, o uso dessas espécies em sistemas produtivos e, ou, em programas de recuperação e conservação ambiental, especialmente em solos de baixa fertilidade, requer estudos futuros sobre os requerimentos nutricionais das espécies. A distinção nutricional entre as espécies pode estar associada a diversos fatores, como a baixa taxa de crescimento e consequente redução de requerimento de nutrientes, o grau de desenvolvimento do sistema radicular e sua atividade fisiológica, a dependência micorrízica e a bactérias diazotróficas, a alta taxa de retranslocação e utilização de nutrientes (Chapin et al., 1983; Resende et al., 1999; Gama-Rodrigues et al., 2008).

Em relação ao teor de nutrientes na folha, a dispersão gráfica das espécies (Figura 2) demonstra dissimilaridade entre elas em cada fragmento florestal, e entre ambos os fragmentos. $\mathrm{O} \mathrm{P}$ foi a variável mais estreitamente associada (carga relativa $=-0,782$ ) ao primeiro componente principal (Figura 2a). Isso indica que esse nutriente foi a principal variável na distinção das espécies no fragmento M1. Os demais nutrientes foram de pequeno significado $(<0,70)$ na distinção das espécies. Por outro lado, no fragmento M2 o K foi a variável que mais contribuiu na dissimilaridade entre as espécies (carga relativa $=-0,835)$, tendo os outros nutrientes pouco significado $(<0,70)$ na distinção das espécies (Figura 2b). Na análise conjunta dos dois fragmentos florestais, o K (carga $=0,869)$ e o $\mathrm{P}$ (carga $=0,805$ ) foram as principais variáveis na dissimilaridade entre as espécies (Figura 2c). Houve nítida distinção das espécies entre os dois fragmentos florestais (Figura 2c). Praticamente todas as espécies do fragmento M1 ficaram concentradas à direita nos quadrantes superior e inferior, ao contrário das espécies do fragmento M2, que se posicionaram à esquerda do quadrante. Esses resultados mostram o potencial de uso de componentes principais como método de análise para separar sítios florestais para um futuro manejo, com base na avaliação nutricional das espécies. Seriam necessários estudos com maior número de fragmentos florestais para aprimorar esse método de análise.

O teor médio de nutrientes em galhos das árvores do M1 foi de 8,55, 0,41, 2,41, 2,96 e 1,46 $\mathrm{g} \mathrm{kg}^{-1}$ de N, $\mathrm{P}, \mathrm{K}, \mathrm{Ca}$ e $\mathrm{Mg}$, respectivamente. Já no M2, o teor médio de nutrientes em galhos foi de 6,16, 0,45, 2,75, 4,52 e 1,54 g kg-1 de N, P, K, Ca e Mg, respectivamente. Esses resultados indicam que, na copa das árvores de ambos os fragmentos florestais, a maior concentração de nutrientes ocorreu nas folhas. Em geral, os teores médios de nutrientes são maiores nas folhas do que em galhos, à exceção do Ca (Drumond, 1996; Hughes et al., 1999; Gama-Rodrigues \& Barros, 2002; GamaRodrigues et al., 2007). 
Quadro 3. Teor de nutrientes e carbono em folhas de espécies encontradas no fragmento florestal M1 e M2 da Mata Atlântica, na região norte-fluminense

\begin{tabular}{|c|c|c|c|c|c|c|c|}
\hline \multirow{2}{*}{ Família } & \multirow{2}{*}{ Espécie } & \multicolumn{6}{|c|}{ Folha } \\
\hline & & $\mathbf{N}$ & $\mathbf{P}$ & $\mathbf{K}$ & $\mathbf{C a}$ & Mg & $\mathbf{C}$ \\
\hline & & & & g & & & \\
\hline Euphorbiaceae & Alchornea glandulosa & 22,10 & 1,95 & 18,90 & 9,23 & 2,90 & 448 \\
\hline Annonaceae & Annona cacans & 19,80 & 1,99 & 22,66 & 8,96 & 1,98 & 448 \\
\hline Anacardiaceae & Astronium sp. & 23,10 & 1,54 & 21,56 & 12,30 & 2,23 & 447 \\
\hline Rubiaceae & Bathysa meridionalis & 25,10 & 2,01 & 21,36 & 11,20 & 2,58 & 447 \\
\hline Malphighiaceae & Byrsonima sp. & 22,40 & 2,07 & 24,78 & 8,99 & 3,65 & 440 \\
\hline Meliaceae & Cabralea canjerana & 18,70 & 1,98 & 23,65 & 11,23 & 2,01 & 449 \\
\hline Sapindaceae & Cupania vernalis & 13,60 & 1,23 & 16,25 & 10,25 & 2,87 & 447 \\
\hline Meliaceae & Guarea guidonia & 25,57 & 2,13 & 15,54 & 5,80 & 3,05 & 435 \\
\hline Euphorbiaceae & Hyeronima alchorneoides & 27,47 & 2,51 & 19,53 & 8,79 & 1,95 & 440 \\
\hline Sabiaceae & Meliosma sellowii & 20,30 & 2,13 & 10,22 & 7,18 & 1,69 & 428 \\
\hline Melastomataceae & Miconia cinnamonifolia & 25,60 & 1,09 & 15,48 & 10,56 & 2,58 & 443 \\
\hline Caesalpinoideae & Moldenhawera floribunda & 29,80 & 2,01 & 15,29 & 10,25 & 3,25 & 447 \\
\hline Lauraceae & Nectandra sp. & 37,10 & 1,22 & 25,62 & 9,52 & 2,56 & 440 \\
\hline Lauraceae & Ocotea $\mathrm{sp}$ & 36,90 & 2,21 & 12,93 & 5,93 & 3,03 & 438 \\
\hline Phytolaccaceae & Phytolacca dioica & 43,40 & 1,85 & 16,05 & 10,45 & 5,45 & 446 \\
\hline Myrtaceae & Psidium cattleianum & 19,90 & 1,45 & 14,87 & 10,59 & 2,89 & 447 \\
\hline Caesalpinoideae & Sclerolobium denudatun & 37,50 & 2,15 & 19,89 & 13,25 & 3,62 & 438 \\
\hline Moraceae & Sorocea bonplandii & 29,90 & 2,83 & 24,15 & 15,58 & 6,40 & 441 \\
\hline Melastomataceae & Tibouchina granulosa & 25,60 & 1,26 & 19,89 & 12,30 & 3,98 & 442 \\
\hline Miristicaceae & Virola oleifera & 21,20 & 2,10 & 26,85 & 12,00 & 2,98 & 449 \\
\hline Vochysiaceae & Vochisia tucanorum & 24,60 & 2,01 & 20,58 & 9,89 & 3,08 & 440 \\
\hline Annonaceae & Xylopia sp. & 19,60 & 1,51 & 17,64 & 9,08 & 3,58 & 432 \\
\hline Rutaceae & Zanthoxylum rhoifolium & 28,70 & 1,54 & 17,45 & 6,23 & 3,65 & 441 \\
\hline Myrtaceae & Não identificada & 21,40 & 1,84 & 13,69 & 8,29 & 3,56 & 447 \\
\hline Annonaceae & Não identificada & 20,11 & 0,98 & 12,25 & 13,28 & 2,03 & 448 \\
\hline Lauraceae & Não identificada & 19,20 & 1,78 & 10,26 & 13,27 & 1,98 & 447 \\
\hline Lauraceae & Não identificada & 18,78 & 1,45 & 8,65 & 19,56 & 4,29 & 447 \\
\hline Lauraceae & Não identificada & 27,21 & 1,58 & 9,56 & 12,45 & 2,78 & 425 \\
\hline Euphorbiaceae & Não identificada & 19,78 & 0,99 & 8,74 & 17,52 & 3,56 & 439 \\
\hline Euphorbiaceae & Não identificada & 26,78 & 1,24 & 10,26 & 13,56 & 4,25 & 452 \\
\hline Rubiaceae & Não identificada & 23,00 & 1,32 & 15,26 & 12,87 & 3,21 & 447 \\
\hline Meliaceae & Não identificada & 21,90 & 1,29 & 9,25 & 10,24 & 2,28 & 448 \\
\hline Sapotaceae & Não identificada & 22,87 & 1,45 & 9,14 & 10,56 & 2,25 & 453 \\
\hline Vochyseaceae & Não identificada & 22,89 & 1,47 & 8,75 & 9,25 & 2,36 & 419 \\
\hline Mimosoideae & Anadenathera colubrina & 20,00 & 1,16 & 14,62 & 10,32 & 2,03 & 445 \\
\hline Papilionoideae & Andira fraxinifolia & 26,00 & 1,24 & 7,97 & 8,69 & 0,99 & 420 \\
\hline Caesalpinoideaea & Apuleia leiocarpa & 20,10 & 0,85 & 3,73 & 10,76 & 2,36 & 437 \\
\hline Apocynaceae & Aspidosperma sp. & 22,86 & 1,08 & 10,69 & 7,87 & 3,18 & 449 \\
\hline Palmae & Attalea dubia & 20,10 & 1,23 & 18,23 & 12,78 & 2,69 & 448 \\
\hline Meliaceae & Cabralea canjerana & 30,64 & 0,99 & 4,31 & 10,40 & 1,48 & 436 \\
\hline Myrtaceae & Campomanesia sp. & 24,50 & 1,50 & 14,25 & 8,96 & 3,56 & 448 \\
\hline Flacourtiaceae & Casearia sp. & 27,50 & 0,69 & 8,29 & 16,23 & 1,39 & 448 \\
\hline Lauraceae & Cryptocarya aschersoniana & 20,10 & 0,80 & 4,04 & 7,42 & 1,41 & 446 \\
\hline Sapindaceae & Cupania vernalis & 35,40 & 0,99 & 6,29 & 13,25 & 3,89 & 450 \\
\hline Apocynaceae & Geissospermum laevis & 26,63 & 1,11 & 4,86 & 8,97 & 4,96 & 447 \\
\hline Meliaceae & Guarea kunthiana & 20,70 & 0,85 & 5,11 & 7,76 & 1,51 & 436 \\
\hline Euphorbiaceae & Hyeronima alchorneoides & 38,30 & 1,06 & 3,64 & 11,03 & 1,31 & 440 \\
\hline Bignoniacea & Jacaranda puberula & 26,73 & 0,79 & 6,27 & 8,04 & 2,18 & 432 \\
\hline Euphorbiaceae & Maprounea guianensis & 22,91 & 1,05 & 7,14 & 7,75 & 2,21 & 443 \\
\hline Rutaceae & Metrodorea nigra & 22,10 & 0,59 & 11,23 & 11,98 & 3,28 & 448 \\
\hline Caesalpinoideae & Moldenhawera floribunda & 25,50 & 0,88 & 10,56 & 8,79 & 4,62 & 444 \\
\hline Myrtaceae & Myrciaria sp. & 28,78 & 0,62 & 4,29 & 9,03 & 2,63 & 440 \\
\hline Lauraceae & Nectandra sp. & 19,20 & 1,05 & 4,99 & 11,34 & 0,87 & 434 \\
\hline Anacardiaceae & Não identificada & 27,83 & 1,09 & 19,23 & 6,98 & 2,69 & 448 \\
\hline Euphorbiaceae & Pogonophora schomburgkiana & 23,70 & 0,84 & 4,44 & 8,78 & 2,83 & 452 \\
\hline Euphorbiaceae & Não identificada & 23,69 & 0,89 & 13,59 & 9,56 & 3,98 & 449 \\
\hline Não identificada & Não identificada & 21,10 & 1,19 & 4,24 & 9,98 & 1,41 & 452 \\
\hline Não identificada & Não identificada & 27,40 & 0,94 & 7,89 & 7,98 & 1,92 & 432 \\
\hline Não identificada & Não identificada & 17,40 & 0,76 & 5,68 & 11,90 & 1,32 & 432 \\
\hline Lauraceae & Não identificada & 21,90 & 0,63 & 3,17 & 13,32 & 2,66 & 456 \\
\hline Lauraceae & Não identificada & 15,80 & 0,65 & 5,53 & 11,42 & 2,99 & 446 \\
\hline Melastomataceae & Não identificada & 23,00 & 0,99 & 19,23 & 18,23 & 3,98 & 448 \\
\hline Myrtaceae & Não identificada & 27,20 & 0,78 & 3,27 & 7,91 & 1,67 & 420 \\
\hline Myrtaceae & Não identificada & 22,10 & 0,99 & 2,95 & 9,18 & 4,03 & 432 \\
\hline Euphorbiaceae & Pachystroma longifolium & 34,35 & 0,70 & 3,96 & 8,05 & 2,19 & 448 \\
\hline Phytolaccaceae & Phytolacca dioica & 22,50 & 1,05 & 9,81 & 11,73 & 3,42 & 436 \\
\hline Sapotaceae & Pouteria sp. & 18,60 & 0,75 & 3,51 & 11,21 & 1,75 & 436 \\
\hline Sapotaceae & Pouteria torta & 25,34 & 0,97 & 9,20 & 10,16 & 1,51 & 439 \\
\hline Elaeocarpaceae & Sloanea sp. & 23,00 & 0,99 & 7,38 & 9,29 & 1,22 & 470 \\
\hline Moraceae & Sorocea bonplandii & 20,40 & 0,95 & 12,09 & 7,23 & 3,91 & 435 \\
\hline Annonaceae & Xylopia sp. & 30,40 & 0,81 & 7,02 & 14,57 & 1,61 & 443 \\
\hline Rubiaceae & Não identificada & 23,73 & 0,95 & 5,51 & 9,22 & 3,42 & 440 \\
\hline
\end{tabular}



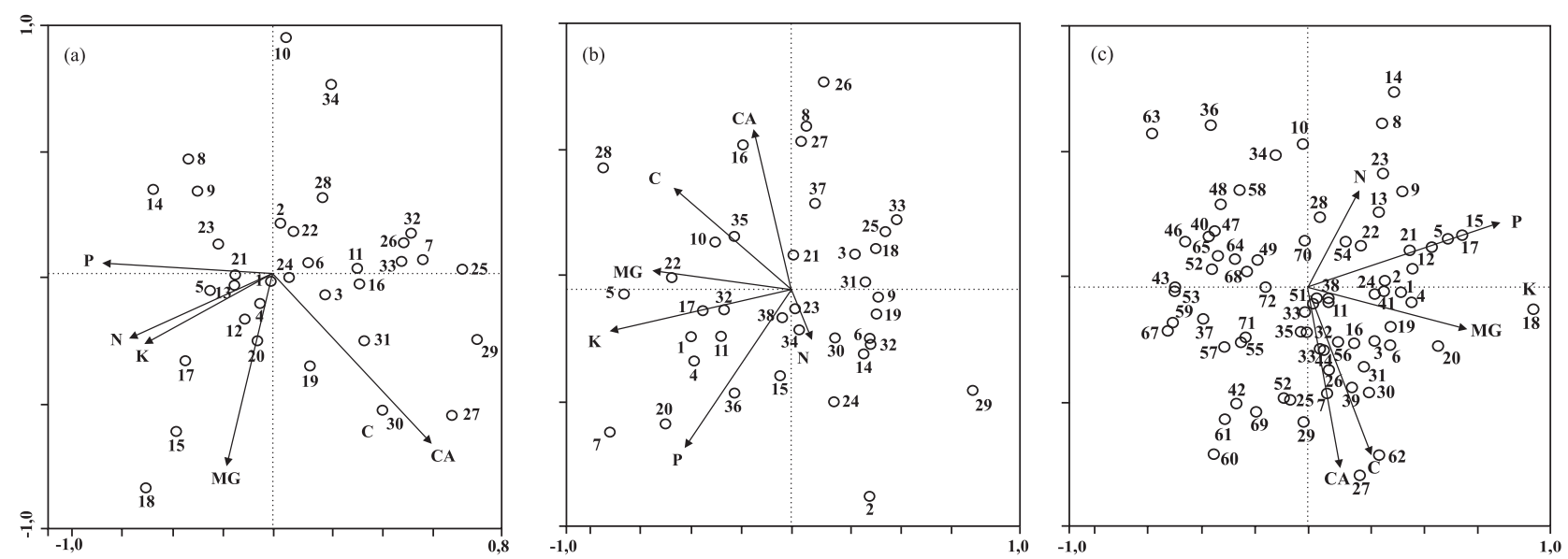

Figura 2. Diagrama de ordenação produzido pela análise de componentes principais do teor de nutrientes e carbono em folhas de espécies encontradas em fragmentos florestais da Mata Atlântica, na região norte-fluminense. (a) fragmento M1; (b) fragmento M2; e (c) fragmentos M1 (espécies de $n^{\circ} 1$ a 34) e M2 (espécies de $n^{0} 35$ a 72 ).

Os teores médios de nutrientes na casca foram bem maiores do que no lenho em ambos os fragmentos florestais (Quadro 4). No fragmento M1, os teores médios de nutrientes no lenho foram de 3,6, 0,18, 1,04, 1,28 e $0,63 \mathrm{~g} \mathrm{~kg}^{-1}$, respectivamente, para $\mathrm{N}, \mathrm{P}, \mathrm{K}, \mathrm{Ca} \mathrm{e}$ Mg; já na casca, na mesma sequência, a magnitude foi de 13,0, 0,22, 2,49, 11,65 e 0,89 $\mathrm{g} \mathrm{kg}^{-1}$ (Quadro 4). No lenho das árvores do fragmento M2, os teores médios foram de 4,2, 0,09, 0,95, 1,36 e 0,24 $\mathrm{g} \mathrm{kg}^{-1}$, para N, P, K, Ca e Mg, respectivamente; já para a casca, os teores de N, P, K, Ca e Mg foram de 13,2, 0,26, $3,00,16,29$ e $1,17 \mathrm{~g} \mathrm{~kg}^{-1}$, respectivamente. De modo geral, houve tendência de as menores classes diamétricas apresentarem maior concentração de nutrientes, tanto no lenho quanto na casca.

Diferenças no teor de nutrientes entre casca e lenho foram relatadas por Clevelario Júnior (1996), Drumond (1996) e Gama-Rodrigues et al. (2007) em florestas da Mata Atlântica. Hughes et al. (1999) encontraram maiores teores de nutrientes nas menores classes diamétricas em florestas naturais do México. Contudo, na maioria dos trabalhos em florestas tropicais os dados de teor de nutrientes referem-se ao tronco como um todo, sem distinguir em casca e lenho (Fassbender, 1993; Hughes et al., 1999; Shanmughavel et al., 2001), e também sem distinção entre diferentes classes de diâmetro (Leão \& Silva, 1991; Fassbender, 1993; Clevelario Júnior, 1996, Drumond et al., 1997; Shanmughavel et al., 2001).

Em ambos os fragmentos florestais estudados, a variação no teor de C foi muito pequena entre as espécies nos componentes da parte aérea das árvores e entre as classes diamétricas (Quadros 3 e 4). Em média, o teor de $\mathrm{C}$ foi de $443 \mathrm{~g} \mathrm{~kg}^{-1}$ (CV 1,9\%) nas folhas, de $453 \mathrm{~g} \mathrm{~kg}^{-1}$ (CV 1,5 \%) no lenho e de $445 \mathrm{~g} \mathrm{~kg}^{-1}$ (CV 1,2 \%) na casca. Esses resultados indicam que o teor de $\mathrm{C}$ seria bastante estável na parte aérea e, por conseguinte, seria a variável que menos contribuiria na dissimilaridade das espécies em relação ao teor de nutrientes (Figura 2).

A sequência decrescente de estoque de nutrientes na parte aérea das árvores nos fragmentos estudados foi de $\mathrm{N}>\mathrm{Ca}>\mathrm{K}>\mathrm{Mg}>\mathrm{P}$ (Quadro 5). Essa mesma sequência também foi observada em outros fragmentos de florestas tropicais (Golley et al., 1978; Brown et al., 1994), em particular da Mata Atlântica (Leão \& Silva, 1991; Clevelario Júnior, 1996; Drumond et al., 1997). No entanto, Gama-Rodrigues et al. (2008) encontraram a sequência de $\mathrm{Ca}>\mathrm{N}>\mathrm{K}>\mathrm{Mg}>\mathrm{P}$ para fragmentos de Mata Atlântica no sudeste da Bahia. Por sua vez, em florestas tropicais da Ásia, a sequência de acúmulo foi de $\mathrm{N}>\mathrm{K}>\mathrm{Mg}>\mathrm{Ca}>\mathrm{P}$ (Shanmughavel et al., 2001). No fragmento M1, o lenho foi o componente de maior estoque de nutrientes, à exceção de Ca. Já no fragmento M2, o galho e o lenho mostraram estoques similares para $\mathrm{P}, \mathrm{K}$ e Mg. Neste fragmento, ainda, o maior estoque de $\mathrm{N}$ ocorreu no lenho e o de Ca na casca (Quadro 5). O fragmento M1 apresentou maior estoque total de N, P e Mg na biomassa aérea do que o M2 (Quadro 6). Os estoques de $\mathrm{N}$ e $\mathrm{P}$ de ambos os fragmentos florestais foram semelhantes aos de outros fragmentos de Mata Atlântica montana (Clevelario Júnior, 1996; Drumond et al., 1997). Entretanto, os estoques de todos os nutrientes, à exceção de $\mathrm{N}$, dos fragmentos estudados foram bem inferiores aos de diversos fragmentos florestais de terras baixas (Brown et al., 1994; GamaRodrigues et al., 2008).

Os resultados do presente estudo evidenciaram que na exploração desses fragmentos florestais seria recomendável a operação de descasque na área, a fim de reduzir a exportação de nutrientes pela retirada da madeira. Também, a não exportação de galhos e folhas contribuiria significativamente para a permanência de quantidades consideráveis de nutrientes na área do fragmento, o que diminuiria a depleção do nível de 
Quadro 4. Teor de nutrientes e carbono no lenho e na casca, em função da classe diamétrica, das árvores amostradas no fragmento florestal M1 e M2 da Mata Atlântica, na região norte-fluminense. Os valores entre parênteses correspondem ao desvio-padrão

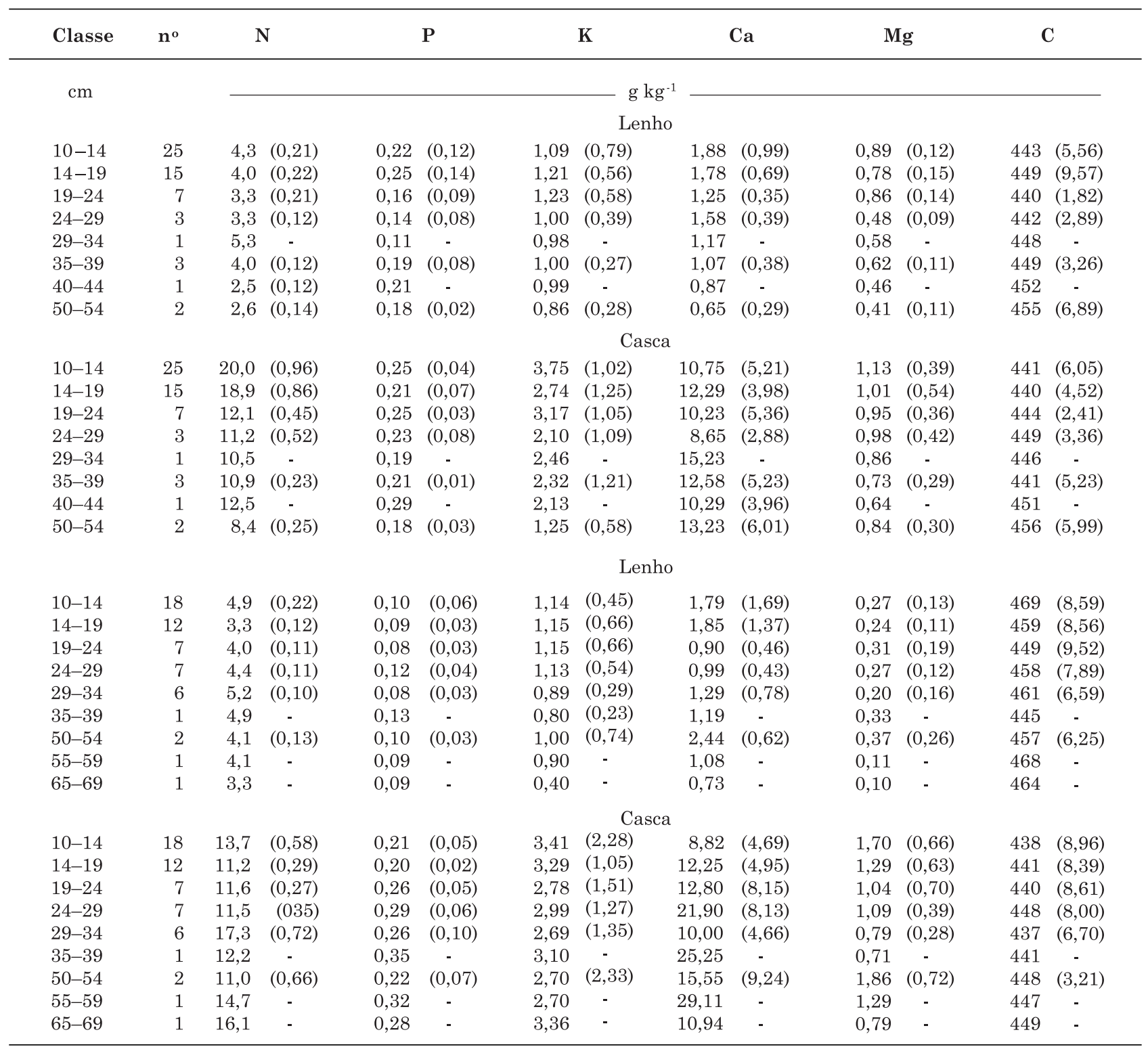

$\mathrm{n}^{0}$ : número de plantas amostradas.

fertilidade dos solos. Esta tem sido a prática recomendada para plantios florestais de eucalipto (Zaia \& Gama-Rodrigues, 2004) e de espécies nativas da Mata Atlântica (Gama-Rodrigues et al., 2007).

Os fragmentos M1 e M2 apresentaram, respectivamente, 65,9 e 68,4 $\mathrm{Mg} \mathrm{ha}^{-1}$ de C na parte aérea (Quadro 6). Em outros fragmentos de Mata Atlântica montana, o estoque de C na biomassa aérea variou de 50 a $165 \mathrm{Mg}$ ha $^{-1}$ (Clevelario Júnior, 1996; Drumond et al., 1997). Em florestas tropicais montanas, o estoque de $\mathrm{C}$ na parte aérea, de maneira geral, varia de 99 a 180 Mg ha-1 (Jordan, 1985; Fassbender, 1993).
A biomassa de serapilheira acumulada no solo dos fragmentos M1 e M2 foi de 8,5 e de 8,8 $\mathrm{Mg} \mathrm{ha}^{-1}$, respectivamente. Em florestas da Mata Atlântica, na Serra do Imbé, região norte-fluminense, a serapilheira acumulada foi de 9,2 $\mathrm{Mg} \mathrm{ha}^{-1}$ (Mazurec, 1998), e em outros fragmentos de Mata Atlântica do tipo montana, também no Estado do Rio de Janeiro, variou de 8,6 a 9,2 $\mathrm{Mg} \mathrm{ha}^{-1}$ (Oliveira \& Lacerda, 1993). Os dois fragmentos florestais estudados praticamente apresentaram os mesmos estoques de $\mathrm{C}$ e de nutrientes na serapilheira (Quadro 6). Entre os nutrientes, o maior estoque foi de $\mathrm{N}$, seguido de $\mathrm{Ca}>\mathrm{Mg}>\mathrm{K}>\mathrm{P}$. 
Quadro 5. Estoque de nutrientes e carbono dos componentes das árvores nos fragmentos florestais M1 e M2 da Mata Atlântica, na região norte-fluminese. Os valores entre parênteses correspondem ao desviopadrão

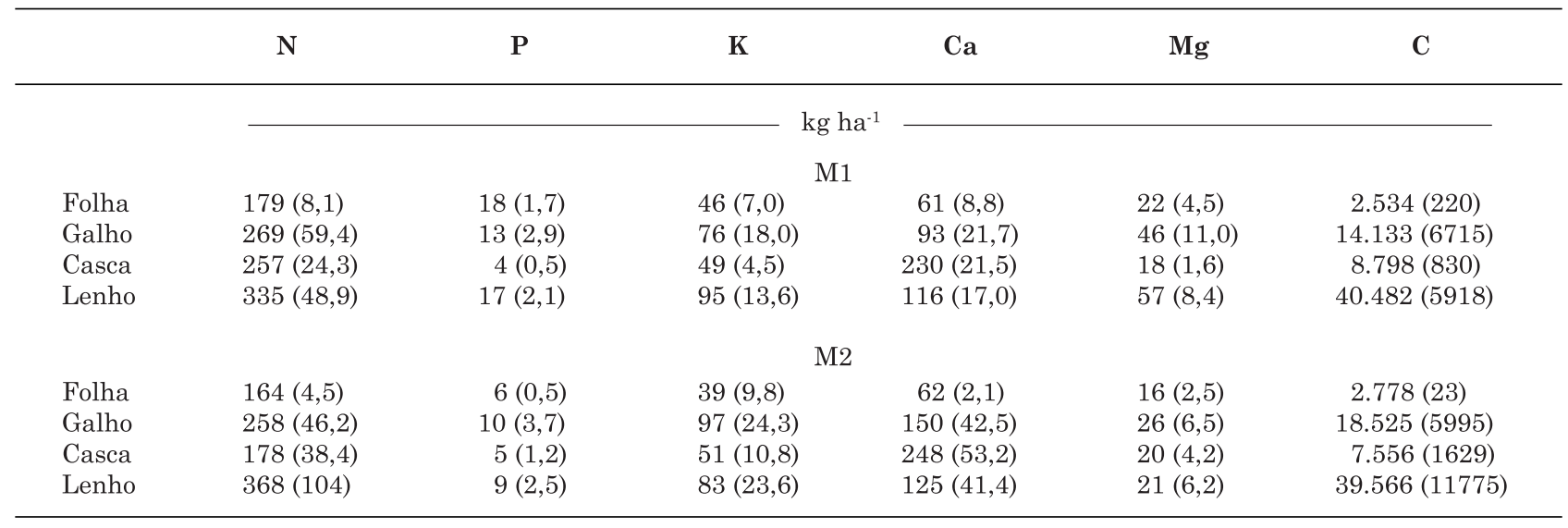

Quadro 6. Carbono e nutrientes na vegetação (parte aérea + serapilheira) e no solo (camada de 0-80 cm), em valores absolutos e percentuais, e balanço nutricional em fragmentos florestais (M1 e M2) da Mata Atlântica, na região norte-fluminense

\begin{tabular}{|c|c|c|c|c|c|c|c|c|}
\hline & \multicolumn{3}{|c|}{ M1 } & \multicolumn{3}{|c|}{ M2 } & \multicolumn{2}{|c|}{ Balanço(1) $^{(1)}$} \\
\hline & $\mathbf{P A}^{(2)}$ & SER & Solo & PA & SER & Solo & M1 & M2 \\
\hline & & & & & $\mathrm{kg} \mathrm{ha}^{-1}$ & & 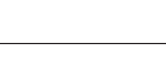 & \\
\hline $\begin{array}{l}\mathrm{C} \\
\%\end{array}$ & $\begin{array}{r}65.947 \\
32,4\end{array}$ & $\begin{array}{r}3.514 \\
1,7\end{array}$ & $\begin{array}{r}133.770 \\
65,8\end{array}$ & $\begin{array}{r}68.425 \\
33,8\end{array}$ & $\begin{array}{r}3.675 \\
1,8\end{array}$ & $\begin{array}{r}130.158 \\
64,4\end{array}$ & +64.309 & +58.058 \\
\hline $\begin{array}{l}\mathrm{N} \\
\%\end{array}$ & $\begin{array}{r}1.040 \\
8,5\end{array}$ & $\begin{array}{r}140 \\
1,1\end{array}$ & $\begin{array}{r}11.122 \\
90,4\end{array}$ & $\begin{array}{l}968 \\
7,7\end{array}$ & $\begin{array}{l}156 \\
1,2\end{array}$ & $\begin{array}{r}11.456 \\
91,1\end{array}$ & +9.942 & +10.332 \\
\hline $\begin{array}{l}\mathrm{P} \\
\%\end{array}$ & $\begin{array}{l}52 \\
58,1\end{array}$ & $\begin{array}{l}3,5 \\
3,9\end{array}$ & $\begin{array}{l}34,0 \\
38,0\end{array}$ & $\begin{array}{l}30 \\
44,8\end{array}$ & $\begin{array}{l}3,3 \\
4,9\end{array}$ & $\begin{array}{r}33,9 \\
50,7\end{array}$ & $-21,5$ & $+0,60$ \\
\hline $\begin{array}{l}\mathrm{K} \\
\%\end{array}$ & $\begin{array}{l}266 \\
59,6\end{array}$ & $\begin{array}{l}9,1 \\
2,0\end{array}$ & $\begin{array}{r}171,4 \\
38,4\end{array}$ & $\begin{array}{l}270 \\
63,1\end{array}$ & $\begin{array}{l}7,8 \\
1,8\end{array}$ & $\begin{array}{r}150,1 \\
35,1\end{array}$ & $-103,7$ & $-127,7$ \\
\hline $\begin{array}{l}\mathrm{Ca} \\
\%\end{array}$ & $\begin{array}{l}500 \\
44,2\end{array}$ & $\begin{array}{r}63,0 \\
5,6\end{array}$ & $\begin{array}{r}568,9 \\
50,3\end{array}$ & $\begin{array}{l}585 \\
57,2\end{array}$ & $\begin{array}{r}59,0 \\
5,8\end{array}$ & $\begin{array}{r}379,2 \\
37,1\end{array}$ & $+5,9$ & $-264,8$ \\
\hline $\begin{array}{l}\mathrm{Mg} \\
\%\end{array}$ & $\begin{array}{c}143 \\
36,8\end{array}$ & $\begin{array}{r}15,4 \\
4,0\end{array}$ & $\begin{array}{r}230,2 \\
59,2\end{array}$ & $\begin{array}{l}83 \\
28,4\end{array}$ & $\begin{array}{r}14,3 \\
4,9\end{array}$ & $\begin{array}{r}194,6 \\
66,7\end{array}$ & $+71,8$ & $+97,3$ \\
\hline
\end{tabular}

${ }^{(1)}$ Balanço de nutrientes: solo - (parte aérea + serapilheira). ${ }^{(2)}$ PA: parte aérea; SER: serapilheira.

Os dois fragmentos florestais praticamente apresentaram o mesmo estoque de $\mathrm{C}$ e nutrientes no perfil de solo amostrado, à exceção do $\mathrm{Ca}$, que no fragmento M1 foi superior em $33 \%$ ao fragmento M2 (Quadro 6).

O estoque total de $\mathrm{C}$ acumulado no sistema solo + vegetação foi aproximadamente de $202,7 \mathrm{Mg} \mathrm{ha}^{-1} \mathrm{em}$ ambos os fragmentos florestais. Os estoques totais de $\mathrm{N}, \mathrm{K}$ e Ca pouco variaram entre os fragmentos. O fragmento M1, contudo, foi superior ao M2 em torno de $25 \%$ para $\mathrm{P}$ e Mg (Quadro 6). Entretanto, no balanço de $\mathrm{C}$ e nutrientes houve distinção entre os fragmentos florestais: no M1, o balanço foi negativo para
$\mathrm{P}$ e K, enquanto no M2 foi negativo para Ke Ca. Além disso, os estoques de $\mathrm{Ca}$ e $\mathrm{P}$ no solo foram de apenas 50,3 e 50,7 \%, respectivamente, nos fragmentos M1 e M2. Balanço negativo indica a baixa capacidade de suprimento de nutrientes do solo para a mesma produção de biomassa em rotações futuras nos fragmentos florestais estudados. Assim, quanto menor a reserva de matéria orgânica e de nutrientes na vegetação no sistema florestal, maior é a estabilidade deste com relação a uma perturbação da vegetação por causas naturais (vento, fogo e tombamento) ou antropológicas (exploração, corte e queima) (Fassbender, 1993). 
Em florestas tropicais montanas, os balanços de C, N, P, Ca e Mg são positivos, mas negativos para K (Grimm \& Fassbender, 1981; Jordan, 1985; Fassbender, 1993). Entretanto, a disponibilidade de N pode ser um fator limitante nesses ecossistemas, pela restrição das taxas de mineralização desse elemento devido às baixas temperaturas (Tanner et al., 1998). Costa (2002), trabalhando também nos dois fragmentos florestais do presente estudo, verificou que a taxa de mineralização de $\mathrm{N}$ em um ano foi inferior a $50 \%$. Hughes et al. (1999) encontraram balanço positivo de C em florestas secundárias tropicais do México. Entretanto, de maneira geral, o balanço de C em florestas naturais de terras baixas nos trópicos úmidos é negativo (Fassbender, 1993; Brown et al., 1994; Gama-Rodrigues et al., 2008), onde, em média, cerca de $60 \%$ do $C$ está imobilizado na biomassa vegetal. No entanto, o balanço de $\mathrm{N}$ total é positivo (Jordan, 1985; Fassbender, 1993; Gama-Rodrigues et al., 2008). Os balanços de P, Ke Ca são, normalmente, negativos, e os de Mg, positivos (Jordan, 1985; Fassbender, 1993; Brown et al., 1994; Gama-Rodrigues et al., 2008).

\section{CONCLUSÕES}

1. As espécies florestais avaliadas nos fragmentos florestais montanos revelaram capacidade diferenciada de acumulação de nutrientes.

2. Os fragmentos florestais montanos na região norte-fluminense apresentaram balanço positivo de $\mathrm{C}$.

3. O balanço negativo de $\mathrm{P}, \mathrm{K}$ e Ca indica que esses elementos foram fatores limitantes ao crescimento dos fragmentos florestais estudados da Mata Atlântica montana, na região norte-fluminense.

\section{AGRADECIMENTOS}

À Fundação de Amparo à Pesquisa do Estado do Rio de Janeiro (FAPERJ) e ao Conselho Nacional de Desenvolvimento Científico e Tecnológico (CNPq), pelo auxílio financeiro para a realização deste trabalho.

\section{LITERATURA CITADA}

ALEGRE, J.; AREVADO, L.; RICSE, A.; CALLO-CONCHA, D. \& PALM, C. Secuestramiento de carbono com sistemas alternativos em el Peru. In: MÜLLER, M.W.; GAMARODRIGUES, A.C.; BRANDÃO, I.C.S.F.L. \& SERÓDIO, M.H.C.F., eds. Sistemas agroflorestais, tendência da agricultura ecológica nos trópicos: Sustento da vida e sustento de vida. Ilhéus, SBSAF/CEPLAC/UENF, 2004. p.27-32

ANDERSON, J.D. \& INGRAM, J.S.I. Tropical soil biology and fertility: A handbook of methods. 2.ed. Wallingford, $\mathrm{CAB}$ International, 1996. 221p.
BARRETO, P.A.; GAMA-RODRIGUES, E.F.; GAMARODRIGUES, A.C. \& BARROS, N.F. \& FONSECA, S. Atividade microbiana, carbono e nitrogênio da biomassa microbiana em plantações de eucalipto, em sequência de idades. R. Bras. Ci. Solo, 32:611-619, 2008.

BATAGLIA, O.C.; FURLANI, A.M.C.; TEIXEIRA, J.P.F.; FURLANI, P.R. \& GALLO, J.R. Métodos de análise química de plantas. Campinas, Instituto Agronômico de Campinas, 1983. 48p. (Boletim Técnico, 78)

BORÉM, R.A.T. Estudo das relações solo x vegetação em topossequências de áreas de domínio da mata atlântica. Campos dos Goytacazes, Universidade Estadual do Norte Fluminense, 1998. 165p. (Tese de Doutorado)

BRAGA, J.M. \& DEFELIPO, B.V. Determinação espectrofotométrica de fósforo em extrato de solo e material vegetal. R. Ceres, 21:73-85, 1974.

BROWN, S.; ANDERSON, J.M.; WOOMER, P.L.; SWIFT, M.J. \& BARRIOS, E. Soil biological processes in tropical ecosystems. In: WOOMER, P.L. \& SWIFT, M.J., eds. The biological management of tropical soil fertility. Nairobi, TSBF, 1994. p.15-46.

BRASIL. Ministério das Minas e Energia. Secretaria Geral. PROJETO RADAMBRASIL. Folhas SF. 23/24. Rio de Janeiro/Vitória. Geologia, geomorfologia, pedologia, vegetação e uso potencial da terra. Rio de Janeiro, 1983. 780p. (Levantamento de Recursos Naturais)

CAMPOS, J.C.C.; LEITE, H.G.; DA SILVA, G.F.; SOARES, C.P.B. \& CARNEIRO, J.A. Estimação de volumes do tronco e da copa de árvores de povoamentos mistos. R. Árvore, 25:471-480, 2001.

CHAPIN, F.S. \& KEDROWSKI, R. Seasonal changes in nitrogen and phosphorus fractions and autumn retranslocation in evergreen and deciduous taiga trees. Ecology, 64:376-391, 1983.

CIDE. Índice de qualidade dos municípios - verde: IQM - Verde. Rio de Janeiro, Fundação CIDE, RESCANF/UENF, UERJ, IEF, Programa Mata Atlântica, Jardim Botânico do Rio de Janeiro e Associação Mico Leão Dourado, 2004. CD ROM.

CLEVELARIO JUNIOR, J. Distribuição de carbono e elementos minerais em um ecossistema de floresta tropical úmido baixo montano. Viçosa, MG, Universidade Federal de Viçosa, 1996. 150p. (Tese de Doutorado)

COSTA, G.S. Decomposição da serapilheira em florestas plantadas e fragmentos da Mata Atlântica na região nortefluminense. Campos dos Goytacazes, Universidade Estadual do Norte Fluminense, 2002. 113p. (Tese de Doutorado)

DRUMOND, M.A. Alterações fitossociológicas e edáficas decorrentes de modificações da cobertura vegetal na Mata Atlântica, região do Médio Rio Doce, MG. Viçosa, MG, Universidade Federal de Viçosa, 1996. 73p. (Tese de Doutorado)

DRUMOND, M.A.; BARROS, N.F.; SOUZA, A.L. \& SILVA, A.F. Distribuição de biomassa e de nutrientes em diferentes coberturas florestais e pastagem na região do Médio Rio Doce-MG. R. Árvore, 21:187-199, 1997. 
EMPRESA BRASILEIRA DE PESQUISA AGROPECUÁRIA EMBRAPA. Centro Nacional de Pesquisa de Solos. Manual de métodos de análise de solo. 2.ed. Rio de Janeiro, 1997. 212p.

EMPRESA BRASILEIRA DE PESQUISA AGROPECUÁRIA EMBRAPA. Centro Nacional de Pesquisa de Solos. Sistemas brasileiro de classificação de solos. Brasília, Embrapa Produção de Informação, 1999. 412p.

FASSBENDER, H.W. Modelos edafologicos de sistemas agroforestales. Turrialba, CATIE, 1993. 491p.

FERNANDES, E.C.M. Agroforestry for productive and sustainable landscapes in the face of global change. In: GAMA-RODRIGUES, A.C.; BARROS, N.F.; GAMARODRIGUES, E.F.; FREITAS, M.S.M.; VIANA, A.P.; JASMIN, J.M.; MARCIANO, C.R. \& CARNEIRO, J.G.A., eds. Sistemas agroflorestais: Bases científicas para o desenvolvimento sustentável. Campos dos Goytacazes, Universidade Estadual do Norte Fluminense Darcy Ribeiro, 2006. p.15-31.

GAMA-RODRIGUES, A.C. \& MAY, P. Sistemas agroflorestais e o planejamento do uso da terra: Experiência na região norte-fluminense, RJ. In: MACÊDO, J.L.V.; WANDELLI, E.V. \& SILVA JÚNIOR, J.P., eds. Sistemas agroflorestais: Manejando a biodiversidade e compondo a paisagem rural. Manaus, Embrapa, 2001. p.130-136.

GAMA-RODRIGUES, A.C. \& BARROS, N.F. Ciclagem de nutrientes em floresta natural e em plantios de eucalipto e de dandá no sudeste da Bahia, Brasil. R. Árvore, 26:193207, 2002.

GAMA-RODRIGUES, A.C.; BARROS, N.F. \& COMERFORD, N.B. Biomass and nutrient cycling in pure and mixed stands of native tree species in Southeastern Bahia, Brazil. R. Bras. Ci. Solo, 31:287-298, 2007.

GAMA-RODRIGUES, A.C.; GAMA-RODRIGUES, E. \& BARROS, N.F. Balanço de carbono e nutrientes em plantio puro e misto de espécies florestais nativas no sudeste da Bahia. R. Bras. Ci. Solo, 32:1165-1179, 2008.

GAMA-RODRIGUES, A.C. Ciclagem de nutrientes por especies florestais em povoamentos puros e mistos em solos de tabuleiro da Bahia, Brasil. Vicosa, UFV, 1997. 107p. (Dissertacao de Doutorado)

GOLLEY, F.B.; MCGINNIS, J.T.; CLEMENTS, R.G.; CHILD, G.I. \& DUEVER, M.J. Ciclagem de minerais em um ecossistema de floresta tropical úmida. São Paulo, EPUEDUSP, 1978. 256p.

GOMES, J.B.V.; CURI, N.; MOTTA, P.E.F.; KER, J.C.; MARQUES, J.J.G.S.M. \& SCHULZE, D.G. Análise de componentes principais de atributos físicos, químicos e mineralógicos de solos do bioma Cerrado. R. Bras.Ci. Solo, 28:137-154, 2004.

GRIMM, U. \& FASSBENDER, H.W. Ciclos biogeoquímicos en un ecossistema forestal de los Andes Occidentales de Venezuela. I. Inventario de las reservas orgánicas y minerales ( $, \mathrm{P}, \mathrm{K}, \mathrm{Ca}, \mathrm{Mg}, \mathrm{Mn}, \mathrm{Fe}, \mathrm{Al}, \mathrm{Na})$. Turrialba, 31:27-37, 1981.
GRUBB, P.J. Control of forest growth and distribution on wet tropical mountains: With special reference to mineral nutrition. Ann. Rev. Ecol. Syst., 8:83-107, 1977.

HUGHES, R.F.; KAUFFMAN, J.B. \& JARAMILLO, V.J. Biomass, carbon and nutrient dynamics of secondary forest in a humid tropical region of Mexico. Ecology, 80:1892-1907, 1999.

JORDAN, C.F. Nutrient cycling in tropical forest ecosystems. Georgia, John Wiley \& Sons, 1985. 190p.

LEÃO, A.C. \& SILVA, L.A.M. Bioelementos na cobertura vegetal e no solo do ecossistema dos tabuleiros costeiros do sudeste da Bahia, Brasil. Agrotrópica, 3:87-92, 1991.

LUGO, A.E.; CUEVAS, E. \& SANCHEZ, M.J. Nutrients and mass in litter and soil of ten tropical tree plantations. Plant Soil, 125:263-280, 1990.

MAZUREC, A.P. Produção, aporte de nutrientes e decomposição da serrapilheira em Mata Atlântica de encosta em duas altitudes, na Serra do Imbé, Norte Fluminense. Campos dos Goytacazes, Universidade Estadual do Norte Fluminense, 1998. 90p. (Tese de Doutorado)

MORENO, M.T. Estrutura e composição florística do estrato arbóreo em duas zonas altitudinais na Mata Atlântica de encosta da região do Imbé, RJ. Campos dos Goytacazes, Universidade Estadual do Norte Fluminense, 1998. 100p. (Tese de Mestrado)

OLIVEIRA, R.R. \& LACERDA, L.D. Produção e composição química da serrapilheira na floresta da Tijuca (RJ). R.Bras. Bot., 16:93-99, 1993.

RESENDE, A.V.; FURTINI NETO, A.E.; MUNIZ, J.A.; CURI, N. \& FAQUIN, V. Crescimento inicial de espécies florestais de diferentes grupos sucessionais em resposta a doses de fósforo. Pesq. Agropec. Bras., 34:2071-2081, 1999.

SHANMUGHAVEL, P.; LIQING, S.; ZHENG, Z. \& MIN, C. Nutrient cycling in a tropical seasonal rain forest of Xishuangbanna, Southwest China. Part 1: Tree species: Nutrient distribution and uptake. Biores. Technol., 80:163170, 2001.

SILVA, G.C. \& NASCIMENTO, M.T. Fitossociologia de um remanescente de mata sobre tabuleiros no norte do estado do Rio de Janeiro (Mata do Carvão). R. Bras. Bot., 24:5162, 2001.

TANNER, E.V.J.; VITOUSEK, P.M. \& CUEVAS, E. Experimental investigation of nutrient limitation of forest growth on wet tropical mountains. Ecology, 79:10-22, 1998.

TER BRAAK, C.J.F. Canonical correspondence analysis: A new eigenvector technique for multivariate direct gradient analysis. Ecology, 67:1167-1179, 1986.

WICK, B.; TIESSEN, H. \& MENEZES, R. Land use changes following the conversion of the natural vegetation into silvo-pastoral systems in semi-arid NE Brazil. Plant Soil, 222:59-70, 1998.

ZAIA, F.A. \& GAMA-RODRIGUES, A.C. Ciclagem e balanço de nutrientes em povoamentos de eucalipto na região norte-fluminense. R. Bras. Ci. Solo, 28:843-852, 2004. 